\title{
Multifidelity Uncertainty Quantification of a Commercial Supersonic Transport
}

\author{
Thomas K. West IV* and Ben D. Phillips ${ }^{\dagger}$ \\ NASA Langley Research Center, Hampton, VA, 23681
}

The objective of this work was to develop a multifidelity uncertainty quantification approach for efficient analysis of a commercial supersonic transport concept. An approach based on point-collocation, non-intrusive polynomial chaos was formulated in which a low-fidelity model could be corrected using multiple higher-fidelity models. The formulation and methodology also allows for the addition of uncertainty sources not present in the lower fidelity models. To demonstrate the applicability and potential computational savings of the multifidelity polynomial chaos approach, two model problems were explored. The first was a supersonic airfoil with three levels of modeling fidelity, each capturing a gradual increase in modeling of the underlying flow physics. As much as $\mathbf{5 0 \%}$ computational cost reduction was observed using the mutlifidelity approach, while predicting nearly the same amount of uncertainty in drag. The second problem was a commercial supersonic transport. This model had three levels of fidelity that included two different modeling approaches and the addition of physics between the fidelity levels. Results of this analysis yielded nearly a 70\% computational savings to predict a comparable amount of uncertainty in ground noise. Both problems illustrate the applicability and significant computational savings of the multifidelity method for efficient and accurate uncertainty quantification.

\section{Nomenclature}

\begin{tabular}{|c|c|c|c|}
\hline$C$ & Additive Correction & $\alpha$ & Polynomial Chaos Expansion Coefficient \\
\hline$N_{s}$ & Number of Samples & $\kappa$ & von Karman's Constant \\
\hline$N_{t}$ & Number of Terms in a Total-Order & $\Psi$ & Random Basis Function \\
\hline & Polynomial Chaos Expansion & $\phi$ & Model Roll Angle \\
\hline$n$ & Number of Random Dimensions & $\sigma$ & Diffusion Coefficient \\
\hline$q$ & Polynomial Order & $\xi$ & Standard Input Random Variable \\
\hline$R$ & Stochastic Response & & \\
\hline$r$ & Polynomial Order of the Correction & & \\
\hline
\end{tabular}




\section{Introduction}

One of the most substantial obstacles to commercial supersonic flight over land is the adverse impact of a sonic boom. Current Federal Aviation Administration and Committee on Aviation Environmental Protection restrictions prohibit commercial, supersonic flight over many countries, including the United States due to the ground level noise. Recent thrusts in research have focused on reducing the ground noise in hopes of reversing the regulations, in turn increasing the viability of commercial, supersonic flight. In 2003, after a series of tests that measures the ground signature of a modified F-5E supersonic aircraft, the Shape Sonic Boom Demonstrator (SSBD) program, as the concept was named, proved via flight testing that sonic boom mitigation was possible through shape optimization [1]. This has launched programs focusing on the design of low-boom aircraft. Some examples are Gulfstream's Quiet Spike, Lockheed Martin's and Boeing's $N+1$ and $N+2$ supersonic designs, and Japan Aerospace Exploration Agency's (JAXA) NEXST and S3TD programs [2-5]. In 2008, NASA held a Sonic Boom Prediction Workshop to assess the performance of computational fluid dynamics (CFD) for sonic boom prediction[6]. AIAA then hosted the first Sonic Boom Prediction workshop in 2014 [7] and a second one in 2017 [8].

As a means of aiding and accelerating the design process, CFD has become an important part of designing supersonic aircraft. Current state of the art predictions use three-dimensional CFD solvers to determine the near-field pressure signature, which is then numerically propagated to the ground. These numerical results are often used to help inform the design of supersonic vehicles through various techniques, such as adjoint-based optimization employing near-field targeting and ground signature loudness [9-11] and less computationally demanding, derivative-free techniques [12]. These computational models take one set of inputs (which may include the geometry, freestream conditions, angle of attack, or modeling parameters for turbulence and boom propagation) and produce a deterministic set of outputs (e.g., ground signature, final optimized geometry, and near-field flow information). Often, the input parameters may not be known exactly due to inherent variations in flight conditions and manufacturing tolerances. Additionally, error is introduced through assumptions made when creating the relevant mathematical models. The ground noise generated by supersonic aircraft is sensitive to these changes and this motivates the development of a framework which can account for and efficiently represent uncertainties in the design space for use in reliable and robust design of supersonic, low-boom configurations that will meet necessary or prescribed performance standards.

One significant challenge is the efficient propagation of uncertainty through the computational models. This is particularly an issue as more detailed analysis requires models of higher fidelity and more analyses are coupled in a multidisciplinary environment [13]. These models may have a significant amount of uncertainty and propagating it can be challenging even by using recent approaches for improved efficiency [14]. However, many of these uncertainties are common to models of lower fidelity, though their impact may be different. A desirable uncertainty quantification approach would be one that uses the reduced computational cost of lower fidelity models, and provides the accuracy 
of high-fidelity models. Therefore, the objective of this work is to outline an approach to multifidelity uncertainty modeling.

Previous work by Ng and Eldred [15] and by West and Gumbert [16] used an approach based on non-intrusive polynomial chaos to construct a multifidelity surrogate model from two analysis fidelity levels with the same sources of uncertainty. In the current work, the goal is to extend the approach to allow for different uncertainty sources in each fidelity level and to create a multifidelity model from more than two analysis fidelity levels. Additionally, a point-collocation approach is used to determine the multifidelity polynomial chaos expansion coefficients instead of a spectral projection based approach used by $\mathrm{Ng}$ and Eldred [15] to make the use of polynomial chaos more tractable to higher dimension problems. This approach is then applied to the analysis of a current low-boom configuration of interest to quantify the uncertainty in ground noise predictions.

The next section describes the sources of uncertainty in multifidelity modeling. Section III describes the approach for multifidelity surrogate modeling using non-intrusive polynomial chaos. Section IV describes the computational model used in this study and results of a multifidelity uncertainty analysis. The last section outlines and discusses the important conclusions of this work.

\section{Uncertainty in Multifidelity Models}

This section describes the uncertainty that may exist in a multifidelity analysis. A description of the types of uncertainty in numerical modeling is provided, followed by a description of uncertainty in multifidelity problems.

\section{A. Types of Uncertainty in Numerical Modeling}

A critical step in any uncertainty analysis is the classification of uncertain parameters. These parameters may be mathematically represented differently based on the nature of their uncertainty. Incorrect classification and/or treatment of uncertain parameters can result in widely varying output uncertainty.

Two main types of uncertainty exist in numerical modeling: aleatory uncertainty and epistemic uncertainty [17]. Aleatory uncertainty is the inherent variation of a physical system. Such variation is due to the random nature of input data and can be mathematically represented by a probability density function if substantial experimental data is available for estimating the distribution type. An example of this type of uncertainty could be the fluctuations in freestream quantities. While still considered random variables, these variations are not controllable and are sometimes referred to as irreducible uncertainties.

Epistemic uncertainty in a stochastic problem comes from several potential sources. These include a lack of knowledge or incomplete information about the behavior of a particular variable. Also, ignorance or negligence with regards to accurate treatment of model parameters is a source of epistemic uncertainty. In the context of a mathematical model, the assumptions and/or an inadequate knowledge of a physical process lead to what is termed model-form 
uncertainty. Contrary to aleatory uncertainty, epistemic uncertainty is sometimes referred to as reducible uncertainty. An increase in knowledge regarding the physics of a problem, along with accurate modeling, may reduce this type of uncertainty, but typically comes at some cost. Epistemic uncertainty is often modeled using intervals because the use of probabilistic distributions (even a uniform distribution) can lead to inaccurate predictions in the amount of uncertainty in a system. Upper and lower bounds of these intervals can be drawn from limited experimental data or from expert predictions and judgment [18, 19].

An additional, special case of epistemic uncertainty is numerical error. This uncertainty is common in numerical modeling and is defined as a recognizable deficiency in any phase or activity of modeling and simulations that is not due to lack of knowledge of the physical system. In CFD, an example of this type of uncertainty would be the discretization error in both the temporal and spatial domains that comes from the numerical solution of the partial differential equations that govern the system [19]. This uncertainty can be well understood and controlled through code verification and grid convergence studies.

\section{B. Multifidelity Model Uncertainty}

In multifidelity analysis, the objective is to estimate the output of a high-fidelity model by using a combination of low and high-fidelity predictions. When uncertainty is accounted for in the analysis, the term "multifidelity" can have two meanings that need to be investigated, the most common being multifidelity mathematics or physics modeling. The second is multifidelity uncertainty modeling. The latter is important because it can have a different impact on predictions from models of different modeling fidelity and/or models of different fidelity can have different sources of uncertainty.

The real challenge is dealing with this difference in uncertainty. While each fidelity may have a series of input uncertainties which, again, may be the same or different, each model will have a different amount of model-form uncertainty. This is illustrated in Fig. 1 by comparing the sources of uncertainty in low and high-fidelity models to some known truth.

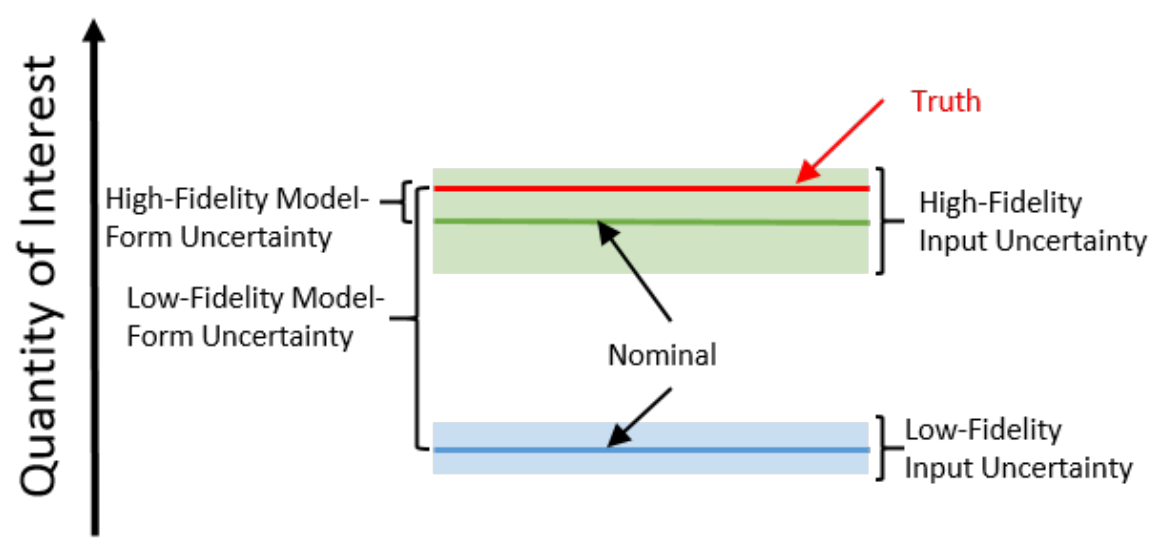

Fig. 1 Example of uncertainty in a multi-fidelity analysis. 
Figure 1 shows that the amount of input uncertainty is larger for the high-fidelity model. In general, this may be expected. Typically, high-fidelity models have more inputs, more tunable parameters and, possibly, more numerical error. Consider a comparison between a CFD model and an engineering correlation. However, regardless of the impact of input uncertainty on each level of fidelity, the model-form uncertainty is going to be greater in lower fidelity models.

The issue with model-form uncertainty is that without some truth (e.g., experimental data), measuring this uncertainty may be challenging or even impossible. In the cases where there is no truth available, the only measure of model-form uncertainty is between the different levels of fidelity, which requires that the high-fidelity model be known as a "better" approximation. When using multifidelity analysis for UQ, accurately quantifying both the input and, if possible, the model-form uncertainties is necessary to ensure accurate uncertainty predictions. This involves quantifying the uncertainty that is common between the levels of fidelity, as well as any uncertainty that is possessed by the different models.

\section{Multifidelity Polynomial Chaos Expansions}

This section outlines the approach for multifidelity modeling and UQ using non-intrusive polynomial chaos. First, generalized polynomial chaos is discussed, followed by a discussion of the point-collocation approach to estimating the expansion coefficients. Then, the multifidelity approach is introduced and a simple example problem is used to demonstrate the applicability.

\section{A. Generalized Polynomial Chaos}

Polynomial chaos is a surrogate modeling technique based on a spectral representation of the uncertainty. An important aspect of spectral representations is the decomposition of a response value or random function $R$ into a linear combination of separable deterministic and stochastic components, as shown in Eq. (1) for a polynomial of order $q$ and dimension $n$.

$$
R(\boldsymbol{D}, \boldsymbol{\xi}) \approx P_{q, n}=\sum_{i=0}^{N_{t}-1} \alpha_{i}(\boldsymbol{D}) \Psi_{i}(\boldsymbol{\xi})
$$

Here, $\alpha_{i}$ is the deterministic component and $\Psi_{i}$ is the random variable basis functions corresponding to the $i^{\text {th }}$ mode. The basis functions, $\Psi_{i}$, of each random variable are determined using the Askey key [20] and are dependent on the distribution of each random variable. The response, $R$, is a function of independent, deterministic variables, $\mathbf{D}$, and $n$ independent, standard random variables, $\boldsymbol{\xi}$. Note that this series is, by definition, an infinite series; however, in practice, it is truncated and a discrete sum is taken over a number of output modes. To form a complete basis or a total order expansion, $N_{t}$ terms are required, which can be computed from Eq. (2) for a polynomial chaos expansion (PCE) of order $q$ and a number of random dimensions or variables, $n$. 


$$
N_{t}=\frac{(n+q) !}{n ! q !}
$$

Further details on polynomial chaos theory are given by Eldred [21] and Ghanem [22].

\section{B. Point-Collocation, Non-Intrusive Polynomial Chaos}

The objective with any PCE method is to determine the expansion coefficients, $\alpha_{i}$. To do this, polynomial chaos methods can be implemented using an intrusive or a non-intrusive approach. While an intrusive method may appear straightforward in theory, for complex problems this process may be time consuming, expensive, and difficult to implement as changing the deterministic model is required [18]. In contrast, the non-intrusive approach can be easily implemented to construct a surrogate model that represents a complex computational simulation, because no modification to the deterministic model is required. The non-intrusive methods require only the response (or sensitivity) [23-25] values at selected sample points to approximate the stochastic response surface.

Several methods have been developed for non-intrusive polynomial chaos (NIPC). Of these, the point-collocation NIPC method has been used extensively in many aerospace simulation and CFD problems [19, 23, 26, 27] for improved computational efficiency and tractability for high-dimension problems over other spectral projection based approaches. The point-collocation method starts with replacing a stochastic response or random function with its PCE by using Eq. (1). Then, $N_{t}$ sample vectors are chosen in random space and the deterministic code is evaluated at these points, which is the left hand side of Eq. (1). Following this, a linear system of $N_{t}$ equations can be formulated and solved for the expansion coefficients of the PCE. This system is shown in Eq. (3).

$$
\left(\begin{array}{c}
R\left(\mathbf{D}, \boldsymbol{\xi}_{0}\right) \\
R\left(\mathbf{D}, \boldsymbol{\xi}_{1}\right) \\
\vdots \\
R\left(\mathbf{D}, \boldsymbol{\xi}_{P}\right)
\end{array}\right)=\left(\begin{array}{cccc}
\Psi_{0}\left(\boldsymbol{\xi}_{0}\right) & \Psi_{1}\left(\boldsymbol{\xi}_{0}\right) & \ldots & \Psi_{P}\left(\boldsymbol{\xi}_{0}\right) \\
\Psi_{0}\left(\boldsymbol{\xi}_{1}\right) & \Psi_{1}\left(\boldsymbol{\xi}_{1}\right) & \ldots & \Psi_{P}\left(\xi_{1}\right) \\
\vdots & \vdots & \ddots & \vdots \\
\Psi_{0}\left(\boldsymbol{\xi}_{P}\right) & \Psi_{1}\left(\boldsymbol{\xi}_{P}\right) & \ldots & \Psi_{P}\left(\xi_{P}\right)
\end{array}\right)\left(\begin{array}{c}
\alpha_{0} \\
\alpha_{1} \\
\vdots \\
\alpha_{P}
\end{array}\right)
$$

Note that for this linear system, $N_{t}$ is the minimum number of deterministic samples required to obtain a direct solution. If more samples are available and are linearly independent, the system is considered overdetermined and can be solved using a least squares approach. The number of samples over the required minimum is represented by the use of an oversampling ratio (OSR), defined as the ratio of number of actual samples to the minimum number required (i.e., $N_{t}$ ). In general, the number of collocation points can be determined by multiplying Eq. (2) by an OSR. Hosder et al. [28] determined that the PCE is dependent on the number of collocation points and an effective OSR of two was sufficient for the stochastic model problems studied. 


\section{Multifidelity Polynomial Chaos Expansions}

In multifidelity analysis, the goal is to predict the output from a high-fidelity model, $R_{H}$, by correcting outputs from a low-fidelity model, $R_{L}$. With polynomial chaos, one approach is to correct a PCE of the low-fidelity model with a minimal amount of training points from the high-fidelity model as described by $\mathrm{Ng}$ and Eldred [15].

One approach to correcting a low-fidelity model is to use an additive correction, as shown in Eq. (4) for a number of random dimensions $n$ and a low-fidelity model PCE, $P_{q, n}$, of order $q$.

$$
R_{H}=R_{L}+C \approx P_{q, n}+C
$$

For simplicity, first assume the low and high-fidelity models have the same $n$ random dimensions. (The relaxation of this assumption is discussed later.) The PCE of the low-fidelity model, $P_{q, n}$, is corrected by adding $C$. Rearranging Eq. (4), the correction term $C$ can be approximated by Eq. (5), which is a PCE of order $r$, where $r<q$. Note that if $r \nless q$, a PCE of order $q$ could be fit to the high-fidelity model, which would defeat the purpose of a multifidelity model.

$$
C=R_{H}-R_{L} \approx P_{r, n}
$$

The differences in Eq. (5) could be computed by using either the exact result from the low-fidelity model or from a PCE, $P_{q, n}$. However, using the PCE of the low-fidelity model could result in propagating any fitting error. Using the formulation in Eq. (5), Eq. (4) can be rewritten in terms of the PCE coefficients and basis functions, as shown in Eq. (6).

$$
R_{H} \approx P_{q, n}+P_{r, n}=\sum_{\beta \in\left\{\beta: \sum_{j=1}^{n} i_{j} \leq r\right\}}\left(\alpha_{L \beta}+\alpha_{C \beta}\right) \Psi_{\beta}(\vec{\xi})+\sum_{\beta \in\left\{\beta: r<\sum_{j=1}^{n} i_{j} \leq q\right\}} \alpha_{L \beta} \Psi_{\beta}(\vec{\xi})
$$

where,

$$
\beta=\left(i_{1}, \ldots, i_{n}\right)
$$

and

$$
i_{k}=0,1,2,3, \ldots \text { for } k=1, \ldots, n
$$

Here, the set $\beta$ is used to identify the order of each variable. For example, $\beta=(1,0,1)$ would indicate the term that is first order in variable one and first order in variable three. To understand this expansion, each term can be looked at individually. The first term on the RHS states that the expansion coefficients of the PCE representing the low-fidelity model, $\alpha_{L}$, are corrected by adding the expansion coefficients, $\alpha_{C}$, of the PCE representing the correction, $C$, to each common term, up to order $r$. The second term leaves the remaining higher order terms unchanged from the original, 
low-fidelity model PCE as there is not enough information to correct terms above order $r$. Recall that the order of the correction is less than the order of the fit for the low-fidelity model.

With this approach, the expectation is that the low-fidelity model is able to capture the underlying trend of the response as a function of the random variables and also that higher order contributions, above that of the correction PCE order, are minimal or at least well described by the low-fidelity model. After constructing this multifidelity surrogate model, the uncertainty in the models can then be efficiently propagated through the surrogate to obtain an output uncertainty estimate.

The approach can be extended by relaxing the previous assumption that the random variables are the same between the low and high-fidelity models, labeled here as $n_{L}$ and $n_{H}$. If the high-fidelity model possesses random variables in addition to those in the low-fidelity model, the correction term must span the domain of $\xi_{H}$ for which $\xi_{L} \subseteq \xi_{H}$. This results in an additional term being added to Eq. (6) that contains the terms in the correction that do not have a like term in the low-fidelity model.

$$
\begin{gathered}
R_{H} \approx P_{q, n}+P_{r, n}=\sum_{\beta \in\left\{\beta: \sum_{j=1}^{n_{L}} i_{j} \leq r\right\}}\left(\alpha_{L \beta}+\alpha_{C \beta}\right) \Psi_{\beta}(\vec{\xi})+\sum_{\substack{n_{L} \\
\beta \in\left\{\beta: r<\sum_{j=1} i_{j} \leq q\right\}}} \alpha_{L \beta} \Psi_{\beta}(\vec{\xi})+ \\
\sum_{\substack{n_{H} \\
\gamma \in\left\{\gamma: \sum_{j=1} i_{j} \leq r\right\}}} \alpha_{C \gamma} \Psi_{\gamma}(\vec{\xi})
\end{gathered}
$$

where,

$$
\beta=\left(i_{1}, \ldots, i_{n L}\right), \quad \gamma=\left(i_{n L+1}, \ldots, i_{n H}\right)
$$

and

$$
i_{k}=0,1,2,3, \ldots \text { for } k=1, \ldots, n_{H}
$$

Careful consideration should be given to the number of samples needed to construct the low-fidelity model PCE and the correction PCE. As previously stated, the order of $C$ should not meet or exceed the order used for $R_{L}$ as this would eliminate the need for a multifidelity model. However, with this dimension expansion, the correction term could be fit to any order in the variables not common between the low and high-fidelity models by adding only enough samples to capture the non-interaction terms. The caution is just noted here that any practitioner should be mindful of the number of samples of each fidelity level as to not waste resources or possibly degrade accuracy.

Up to this point, the multifidelity PCE formulation only allows for two levels of fidelity. In many cases, there may be additional sources of information that could be used to build up the uncertainty model. For example, two fidelities of computational models and test data may be used together. The ideal approach would be to combine all of the information from $m$ fidelity levels into one PCE. While there is likely numerous ways to do this, one approach is to simply use a 
series of corrections with increasing fidelity. As an example, for an analysis with three levels of fidelity, the lowest would be corrected by the middle level and the resulting multifidelity model would be corrected by the highest fidelity level. As mentioned above, careful consideration should be given to the number of runs at each level as to not waste resources but still achieve an acceptable level of accuracy.

\section{Example Problem}

To demonstrate the multifidelity PCE approach, consider the CFD model of drag on a NACA 0012 airfoil in supersonic flow. The Mach number and angle of attack were selected as 1.4 and 1.25 degrees, respectively. The flow was solved by using FUN3D [29] with second order spatial discretization and the Roe flux scheme. Three levels of fidelity are used in the multifidelity analysis. The low-fidelity was an Euler model of the flow and the mid-level of fidelity was a laminar flow model. The high-fidelity model was a fully turbulent solution of the flow field using the Spallart-Allmaras (S-A) turbulence model. The computational time increases by about a factor of two between each fidelity level on the same hardware. Grid dimensions were chosen such that the solutions are independent of the spatial discretization and the wall spacing for the fully turbulent model was chosen such that $\mathrm{y}^{+}$was less than one everywhere.

For qualitative comparison, Mach contours of baseline low and high-fidelity models are shown in Fig. 2 The primary difference between Figs. 2(a) and 2(b) is the presence of the boundary layer on the body, and how the flow is altered down stream. Note that the mid-fidelity, laminar solution gives a similar flow field visual result compared to the fully turbulent solution.

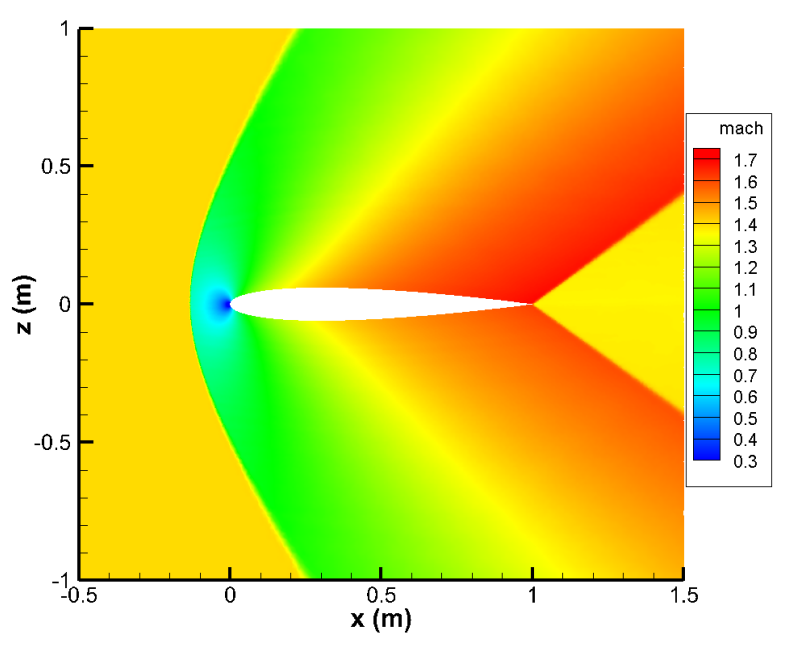

(a) Euler.

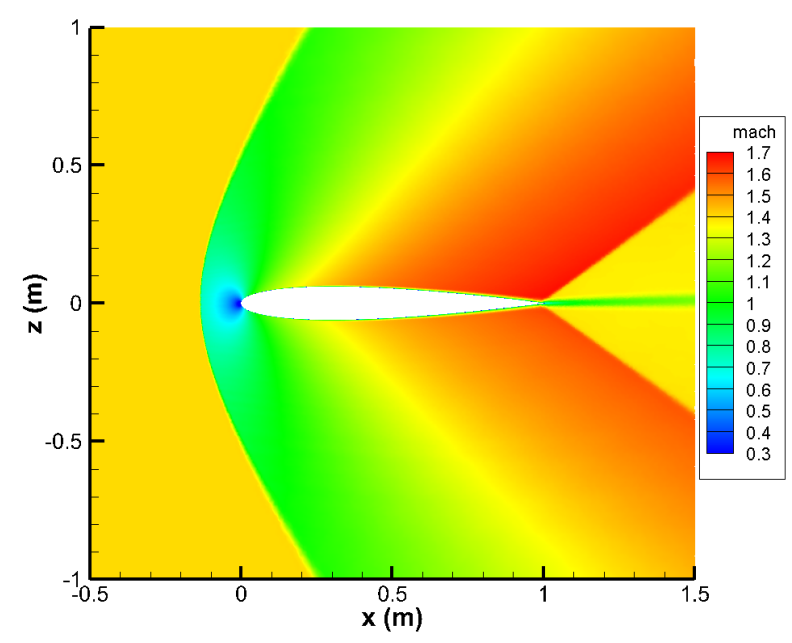

(b) Fully turbulent.

Fig. 2 Mach contours of NACA 0012 flow solution. $M=1.4, \alpha=1.25 \mathrm{deg}$. 
Table 1 Freestream inherent uncertainty sources.

\begin{tabular}{ccc}
\hline \hline Input & Mean & Coefficient of Variance \\
\hline Mach Number & 1.4 & $0.15 \%$ \\
Angle of Attack (deg.) & 1.25 & $5 \%$ \\
\hline
\end{tabular}

Table 2 S-A turbulence model epistemic input uncertainty.

\begin{tabular}{cc}
\hline \hline Input & Interval \\
\hline$\sigma$ (Dissipation coefficient) & {$[0.6,1.0]$} \\
$\kappa$ (von Karman's constant) & {$[0.38,0.42]$} \\
\hline \hline
\end{tabular}

Sources of uncertainty considered in this problem came from inherent variations in Mach number and angle of attack. Both were assumed to be normally distributed with distribution parameters summarized in Table 1 . Note that this uncertainty is consistent with previous work [30].

Additionally, uncertainty in the turbulence model closure coefficients was also considered in the high-fidelity model. Previous works [31-33] have identified that the uncertainty in the von Karman constant $\kappa$ and the $\sigma$ coefficient that governs diffusion in the S-A model contribute significantly to variation in output quantities, such as lift and drag. Uncertainty on these parameters is summarized in Table 2. The details of the turbulence model and uncertainties are left to the references.

To illustrate the difference in output uncertainty and provide a "truth" to compare the multifidelity model, the uncertainties were propagated through the low and high-fidelity models separately. Note that getting a truth using only the high-fidelity model will likely not be possible in practice due to the relatively high computational costs of the high-fidelity model. The nominal drag coefficient values and 95\% confidence intervals are shown in Table 3 . Notice that in addition to the low-fidelity model underpredicting the nominal drag coefficient by about 80 counts, the low-fidelity model underpredicts the amount of uncertainty in the drag coefficient by more than $50 \%$. For this problem, the underprediction is not unexpected as a low-fidelity model is not capturing certain physics of the problem. Note that there is NOT less uncertainty in the low-fidelity model. While Table 3 shows that there is less input uncertainty, the model-form uncertainty is much greater. This is important to understand as ignoring the model-form component could be misleading.

Table 3 Single fidelity drag coefficient predictions.

\begin{tabular}{ccc}
\hline \hline Model Fidelity & Nominal Value & $95 \%$ Confidence Interval \\
\hline Euler & 0.0980 & {$[0.0977,0.0983]$} \\
Fully Turbulent & 0.1062 & {$[0.1052,0.1066]$} \\
\hline \hline
\end{tabular}


Building the three level multifidelity model starts with constructing the low-fidelity PCE. The low-fidelity model has two uncertain parameters. Based on Eq. (2), a second order PCE of drag coefficient would have six terms and would require a minimum of six evaluations of the deterministic model to estimate the expansion coefficients by using a point collocation approach. Because a second order fit is being used for the low-fidelity PCE, there are two choices for the order of the corrections: a constant value (zeroth order) or first order, which would correct the constant and linear terms of the low-fidelity PCE. For this problem, a first order correction using the mid-fidelity model was used and a zeroth order correction was used for the high-fidelity model. A first order correction to the low-fidelity PCE would then require a minimum of three evaluations of the mid-fidelity model. Then, the low and mid multifidelity model can be constructed. Note that the order and number of the correction terms necessary for each level of fidelity is highly problem dependent. Special consideration should be given when making these selections.

To correct this low and mid multifidelity PCE, again, a zeroth order correction of the multifidelity model would require only one sample. However, the high-fidelity model has two additional uncertain parameters that need to be added to the PCE. For these two parameters, first and second order terms were added to the fit, which in total means five evaluations of the high-fidelity model were needed (four for the two new terms and one to update the zeroth order term).

Using the additive approach outlined in the previous section, the multifidelity model can be constructed and the uncertainty can be propagated efficiently through the model by using a Monte Carlo sample approach. The 95\% confidence intervals of the low, high, and multifidelity models are shown in Fig. 3 , where the high-fidelity results were computed for comparison to the other fidelity levels. (Again, in practice, this may not be possible due to the computational expense of the high-fidelity model.) Notice that the multifidelity PCE constructed from the low and mid-fidelity models brings the prediction closer to the high-fidelity model, which is expected because more physics is added to the low-fidelity PCE. However, the added information from the high-fidelity model further improves the accuracy of the multifidelity model. This is a promising result given the significant difference in the physics that are modeled between the three levels of fidelity. Also, the computational savings is significant. A four variable second-order PCE would require a minimum of 15 collocation points, which is what was needed to obtain the high-fidelity only result. The multifidelity model was obtained using six low-fidelity, three mid-fidelity, and five high-fidelity model evaluations. (Recall that the larger high-fidelity sample size is a result of two additional uncertain parameters.) In terms of CPU time, the multifidelity model costs about half the amount of time over the high-fidelity model for nearly the same resulting uncertainty.

\section{Aircraft Model and Computational Analysis Approach}

In this section, the multifidelity PCE approach is applied to the uncertainty analysis of a low-boom supersonic aircraft. First, the Airframe model is described, followed by a discussion of the numerical models used in the multifidelity analysis. Last, the sources of uncertainty are detailed followed by the results of the uncertainty analysis. 


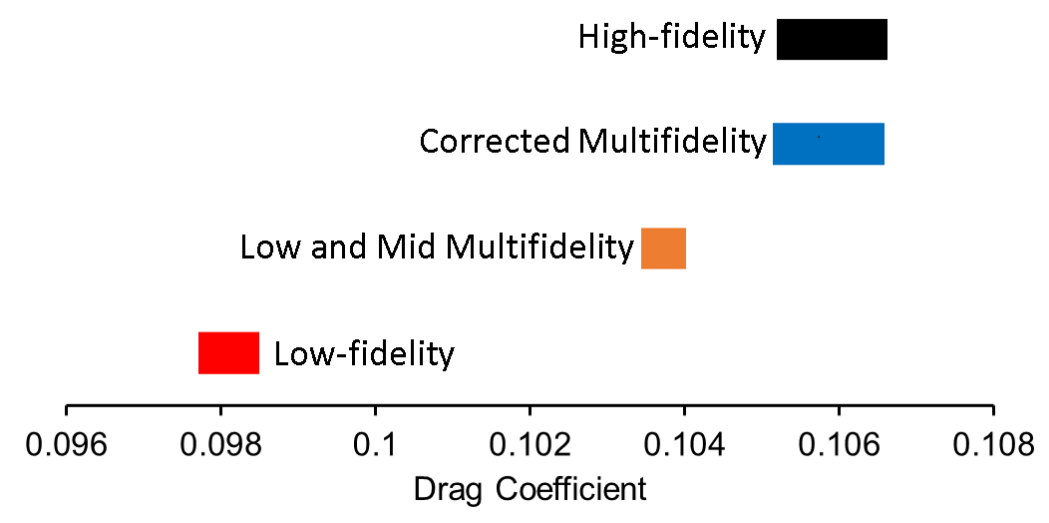

Fig. $395 \%$ confidence intervals of the low, high, and multifidelity models.

\section{A. Airframe Model}

The airframe model that was utilized for a case study in this research is a supersonic aircraft designed as a proof of concept for future full scale low-boom commercial supersonic vehicles. The concept vehicle has a wing span of approximately $30 \mathrm{ft}$, and a reference body length of approximately $90 \mathrm{ft}$. A solid model of the airframe is shown in Fig. 4 .

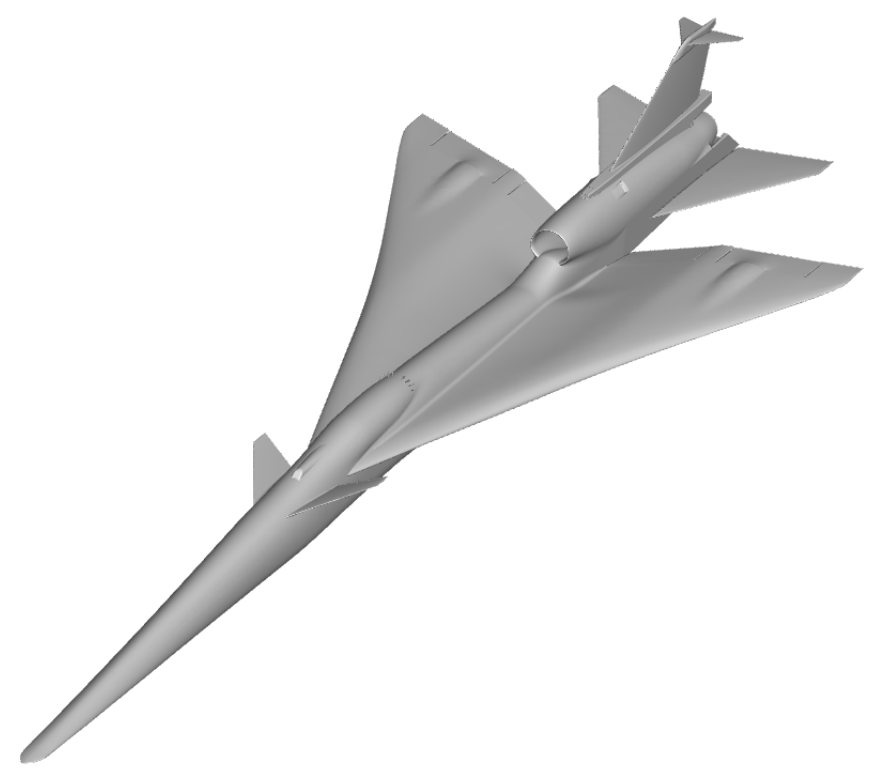

Fig. 4 Airframe model. 


\section{B. Simulation Procedures}

A multifidelity uncertainty analysis of this configuration was conducted with three levels of fidelity. The lowest level is an equivalent area distribution approach [34, 35]. The equivalent area distribution is obtained by computing the surface pressure on the body using, in this work, the inviscid Cart3D solver. This approach is very efficient for predicting the ground signature of a supersonic vehicle compared to running a full off-body boom signature analysis as the computational domain can be significantly reduced. Additionally, this computational model was unpowered (flow-through nacelle). This approach has been used previously in low-boom vehicle, gradient-based design optimization for computational efficiency [36, 37].

The second fidelity level was the Cart3D CFD platform for its established history of predicting near-field sonic boom pressure waveforms [38, 39]. For sonic boom analysis, off-body pressure signatures are extracted from the flowfield solution, which requires the computational domain to span multiple body lengths from the model. Like the low-fidelity model, this computational model was unpowered (flow-through nacelle). The computational domain is adapted by local pressure coefficient to accurately resolve the pressure signature off the body. This approach has been used extensively in low-boom vehicle analysis, design, and previous UQ studies [40, 41].

Lastly, in low-boom vehicle design, effects of boundary layers and shock wave interactions are modeled and utilized to shape the overall signature in fully viscous solvers [14]. Therefore, the highest level of fidelity was a fully-turbulent solution of this configuration using NASA Langley's FUN3D solver [29]. In this model, the Spallart-Allmaras (S-A) turbulence model was used to model the turbulence in the flow field. This model was also fully-powered (i.e., compressor inlet and turbine exit boundary conditions are applied). The computational grid is adapted by Mach number, throughout the domain the resolve the shock structures and the subsequent pressure signature extracted from the flow field.

Once the post-processed, near-field pressure waveforms are generated for each flight condition, the pressure waveforms are passed to an atmospheric propagation code, sBOOM [42]. The propagation code solves an augmented Burgers' equation and simulates how the near-field pressure waveform will change while passing through the atmosphere, to the ground. Running the sBOOM propagation code produces ground signatures (over-pressure vs. time) and performs an integration to produce the A-weighted sound exposure (ASEL) sound levels. The ground signature is then passed to the loudness code [43], which computes perceived loudness, PLdB, and C-weighted sound exposure level, CSEL. This part of the analysis was common to all three fidelity levels of near-field analysis and is relatively computationally inexpensive.

\section{Sources of Uncertainty}

Both the near-field and the atmospheric propagation have uncertain factors which affect the overall prediction of sound at the ground. In the near-field, two uncertain factors were studied, and are listed in Table 4 Each of the factors 
Table 4 Near field CFD aleatory input parameters.

\begin{tabular}{cccc}
\hline \hline Input & Distribution & Mean & Std. Dev. \\
\hline Angle of Attack (deg) & Gaussian & 1.7 & 0.085 \\
Mach Number & Gaussian & 1.42 & 0.002 \\
\hline \hline
\end{tabular}

Table 5 Near-field, CFD S-A turbulence model epistemic input uncertainty.

\begin{tabular}{cc}
\hline \hline Input & Interval \\
\hline$\sigma$ (Dissipation coefficient) & {$[0.6,1.0]$} \\
$\kappa$ (von Karman's constant) & {$[0.38,0.42]$} \\
\hline \hline
\end{tabular}

was characterized as an aleatory uncertainty and assigned a normal distribution. The standard deviations for the Mach number and angle of attack were selected to align with previous UQ for near-field uncertainties [14, 40, 41].

Additionally, uncertainty in the turbulence model closure coefficients for the S-A model was also considered in the high-fidelity model. Previous works [31-33] have identified that the uncertainty in the von Karman constant $\kappa$ and the $\sigma$ coefficient, which governs diffusion, contribute significantly to variation in output quantities. The uncertainty in these parameters is summarized in Table 5 .

Six uncertain factors in the atmospheric propagation were studied. Four of the six factors in the atmospheric propagation were characterized as aleatory uncertainties and are given in Table 6 Each of the factors was assigned a normal distribution.

The relative humidity, temperature, $\mathrm{X}$-wind and $\mathrm{Y}$-wind are represented as deviations from the deterministic profiles and are implemented as a pure bias factor. The deterministic profiles are shown in Fig. 5 , The atmospheric profiles were adopted from the second sonic boom prediction workshop [44] for a case from Green Bay, WI during the winter of 2013. The convention for wind direction for this application is wind in the (positive) X-direction is a tailwind and wind in the Y-direction is cross wind. The uncertainty in the wind profile was determined by information compiled by Schwartz [45], which includes measurement error, as well as temporal and spatial variances.

Table 6 Atmospheric propagation aleatory input parameters.

\begin{tabular}{cccc}
\hline \hline Input & Distribution & Mean & Std. Dev. \\
\hline Relative Humidity & Gaussian & Altitude Dependent & $1 \%$ \\
Temperature & Gaussian & Altitude Dependent & $1 \%$ \\
Xwind & Gaussian & Altitude Dependent & $13.12 \mathrm{ft} / \mathrm{s}$ \\
Ywind & Gaussian & Altitude Dependent & $13.12 \mathrm{ft} / \mathrm{s}$ \\
\hline \hline
\end{tabular}



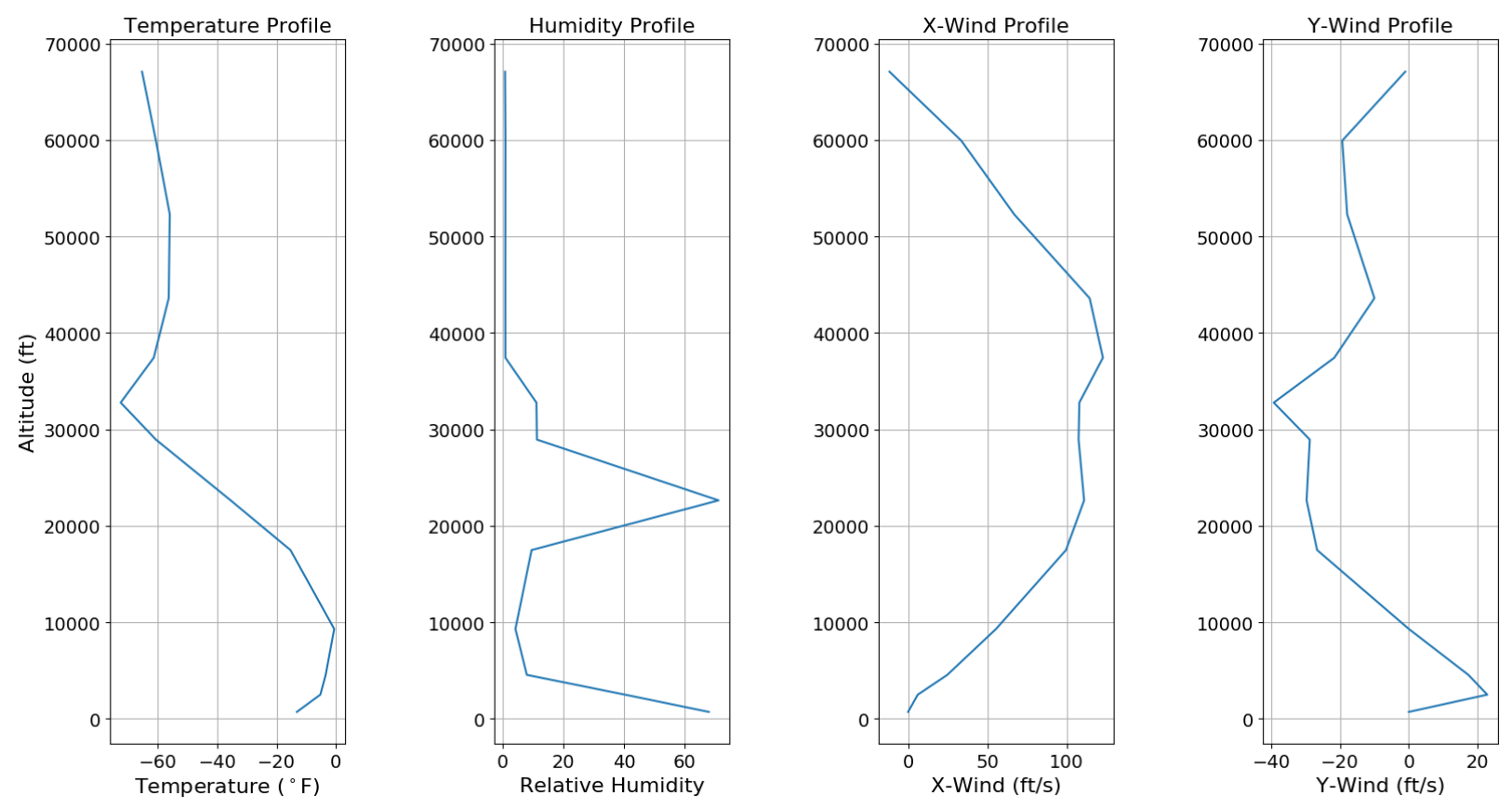

Fig. 5 Deterministic atmospheric profiles.

Table 7 Atmospheric propagation epistemic input parameters.

\begin{tabular}{cc}
\hline \hline Input & Interval \\
\hline Ground Altitude & {$[600,1400 \mathrm{ft}]$} \\
Reflection Factor & {$[1.8,2.0]$} \\
\hline \hline
\end{tabular}

Two of the six uncertain factors in the atmospheric propagation were treated as epistemic uncertainties and are given in Table 7 There is no inference made about the distribution of the epistemic parameters, only a bounding interval is given. The intervals for these factors were chosen to align with previous low-boom UQ work [14, 41].

\section{Baseline Solutions}

Before performing the multifidelity model construction and subsequent uncertainty analysis, baseline solutions of the mid and low-fidelity model were obtained. Mach contours of the symmetry line flow field are shown in Fig. 6 . Notice that most of the shock structure is similar, however, there are some significant differences in the aft portion of the signature due to the engine plume and viscous effects.

Baseline pressure signatures for the mid and high-fidelity models are shown in Fig. 7. Note that there is no baseline signature from the low-fidelity model as there is no generation of an off-body pressure signature. Notice that there are differences in the signatures, particularly in the aft portion of the signature where the powered vehicle produces a different signature as a result of the plume interaction with the flow-field. 


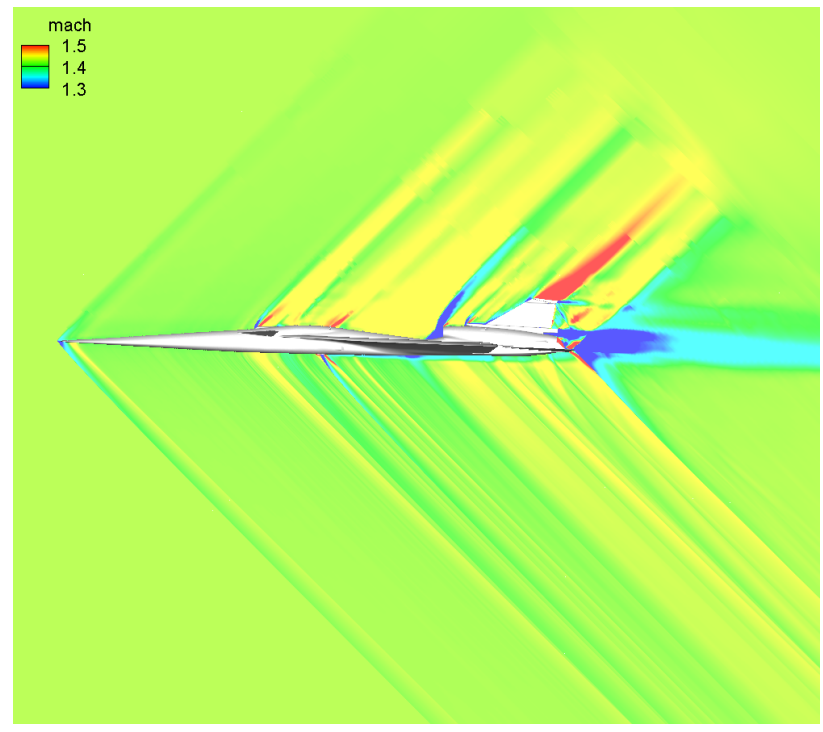

(a) Euler.

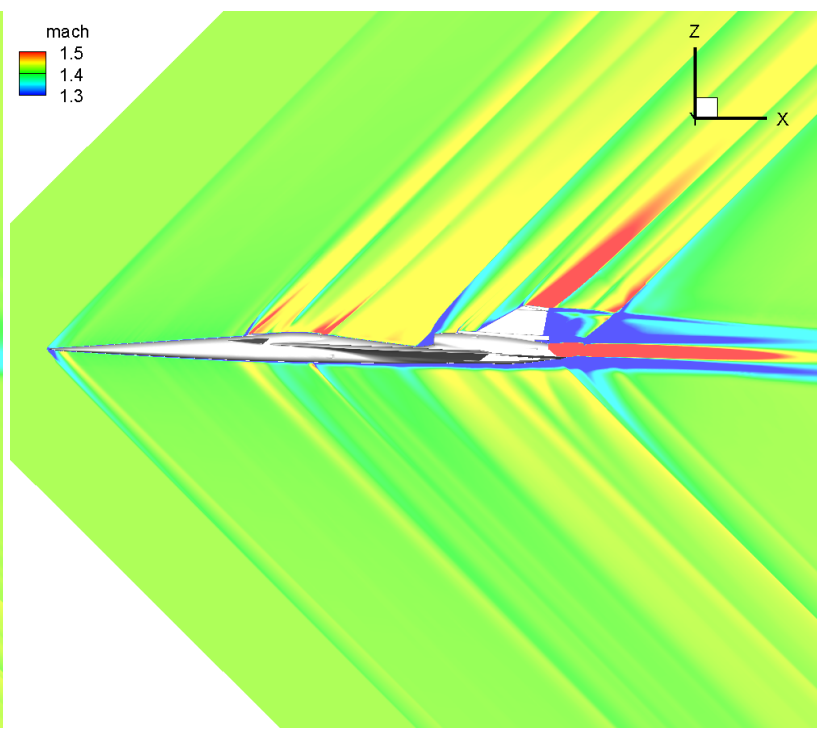

(b) Fully turbulent.

Fig. 6 Baseline Mach contours of the low-boom model.

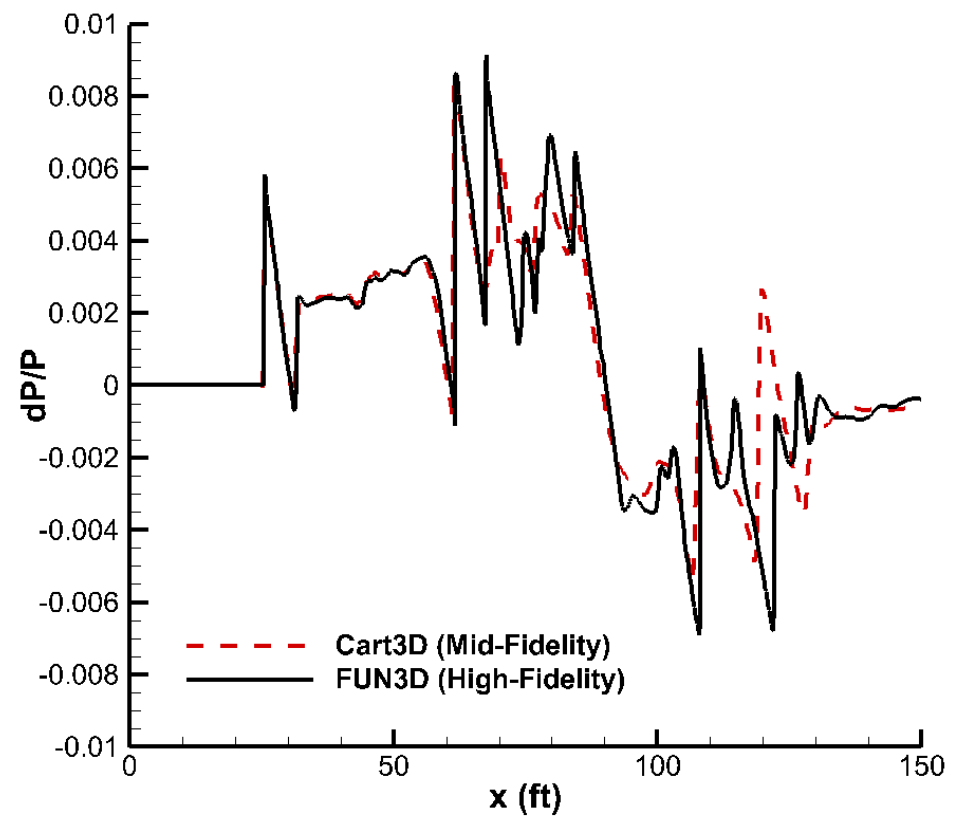

Fig. 7 Baseline near-field pressure signatures.

A summary of the loudness values for each level of fidelity are given in Table 8 . There is a noticeable decrease in loudness as the level of fidelity increases. This is the expected result given how this vehicle was designed with considerations given to shock structure and viscous effects. 


\section{Table 8 PLdB loudness values of the baseline models.}

\begin{tabular}{cc}
\hline \hline Fidelity & PLdB \\
\hline Equivalent Area (low-fidelity) & 80.85 \\
Cart3D Off-Body (mid-fidelity) & 77.72 \\
Fun3D Off-Body (high-fidelity) & 75.13 \\
\hline \hline
\end{tabular}

\section{E. Multifidelity Uncertainty Modeling}

To construct the multifidelity PCE, first, a low-fidelity PCE must be built. There are eight uncertain parameters in the low-fidelity model, which requires a minimum of 45 collocation points for a second-order PCE. Then, the mid-fidelity model was used to correct the low-fidelity PCE. In performing the correction of the low-fidelity model with the mid-fidelity, a significant difference in the dependency on angle of attack with the mid-fidelity was found, which was not found to be significant for the low-fidelity model. Therefore, for the mid-fidelity model, the constant and linear terms of each dimension were corrected in the PCE, along with the second order term for angle of attack. Because the mid-fidelity model has the same eight uncertain parameters, a minimum of 10 samples were needed to correct the low-fidelity model.

Lastly, the low and mid multifidelity PCE needs to be corrected by the high-fidelity model. The high-fidelity model was used to correct the constant (i.e., the mean) of the low and mid multifidelity PCE, as well as the linear term on angle of attack. This again arose due to the dependency of angle of attack in the high-fidelity model that was not captured by even the mid-fidelity model. Additionally, the high-fidelity model has two extra uncertain parameters, namely, the closure coefficients of the S-A turbulence model. First and second-order terms were added to the PCE to capture this uncertainty. In total, six collocation points were needed. The resulting multifidelity PCE model that contains information from all three model fidelity levels could then be used to propagate all of the the uncertainty with a Monte Carlo sampling routine. The resulting 95\% confidence interval of PLdB is shown in Fig. 8 compared to the low and mid multifidelity PCE result and the low-fidelity PCE result.

The results in this figure reflect the information added by each fidelity level. The addition of the mid-fidelity model shifts the mean to the left, but does not add much variance. This is an indication that the low-fidelity model predicts well the underlying trend. The high-fidelity model further shifts the mean, as expected, but there is additional variance added due to the addition of two uncertain parameters.

For verification of the multifidelity model, a full, second-order PCE of the high fidelity model would require at least 76 expensive CFD runs, which was determined to be impractical. Two alternative approaches were used to verify the final multifidelity model. The first was a decoupled uncertainty approach. As described by Phillips and West [40], one approach to reduce the computation burden is to decouple the near-field uncertainty from the propagation uncertainty. In short, the uncertainty in the near-field is computed and used as an input to the uncertainty analysis of the propagation 
Low and Mid Multifidelity

\begin{tabular}{cccccccc}
\multicolumn{10}{c}{ Low-fidelity } & & \\
\hline 70 & 72 & 74 & 76 & 78 & 80 & 82 & 84
\end{tabular}

Fig. $895 \%$ confidence intervals of the low, high, and multifidelity models.

Table 9 Total Sobol indices of key uncertain parameters for PLdB loudness.

\begin{tabular}{cc}
\hline \hline Variable & Sobol Index \\
\hline AoA & 0.24 \\
X-Wind & 0.24 \\
Altitude & 0.15 \\
Reflection Factor & 0.28 \\
$\sigma$ & 0.06 \\
\hline \hline
\end{tabular}

uncertainty, which results in two separate uncertainty analyses. This is an assumption that there is no interaction between the uncertain parameters, but does reduce the computational cost. The result of this decoupled analysis is shown in Fig. 8 There are slight differences in the comparison, but given the assumptions made in both approaches and the significant computational savings of the multifidelity model, the discrepancy was determined to be acceptable.

The second verification approach was to use a series of verification or test points from the high-fidelity model. Ten points, independent from the PCE training data, were used to quantify the error in the multifidelity model. A maximum error of about $0.25 \mathrm{PLdB}$ ( $0.3 \%$ of the baseline loudness) and a median of 0.1 PLdB ( $0.1 \%$ of the baseline loudness) was calculated, both of which are acceptable given the significant computational savings. In total, the above multifidelity model was created at about $30 \%$ of the computational time compared to constructing a full, second-order PCE of the high fidelity model.

As a last step, the sensitivities of the loudness to the uncertain parameters can be determined using Sobol indices based variance decomposition of the PCE [46]. The sensitivities of the important parameters are listed in Table 9 . These results show that much of the uncertainty comes from variation in angle of attack, $\mathrm{X}$-winds, and reflection factor. There is also a small contribution from from the turbulence model parameter $\sigma$. Note that these sensitivities are specific to PLdB. As shown in previous works [14, 40, 41] sensitivities can change with different loudness measures and atmospheric conditions. 


\section{Conclusions}

The objective of this work was to outline an approach to multifidelity uncertainty modeling for efficient and accurate uncertainty quantification of low-boom configurations. A multifidelity surrogate approach based on non-intrusive polynomial chaos was formulated, which allows for the data fusion of any number of fidelity levels into a single, multifidelity model. This resulting model then has the accuracy of a high-fidelity model but required the computational expense of a lower-fidelity model to construct. The approach was applied to two example problems, including an application to low-boom vehicle uncertainty analysis. Results highlight significant computational savings that could be achieved for a similar resulting uncertainty uncertainty prediction. Overall, this approach is very powerful and is well suited for preliminary design and analysis of low-boom supersonic aircraft.

\section{References}

[1] Pawlowski, J., Graham, D., Boccadoro, C., Coen, P., and Maglieri, D., "Origins and Overview of the Shaped Sonic Boom Demonstration Program,” AIAA Paper 2005-0005, 2005.

[2] Howe, D. C., Waithe, K. A., and Haering Jr, E. A., "Quiet Spike Near Field Flight Test Pressure Measurements with Computational Fluid Dynamics Comparisons,” AIAA Paper 2008-0128, 2008.

[3] Morgenstern, J., Norstrud, N., Sokhey, J., Martens, S., and Alonso, J. J., “Advanced Concept Studies for Supersonic Commercial Transports Entering Service in the 2018 to 2020 Period,” NASA CR-2013-217820, NASA Langley Research Center, Feb. 2013. doi:2060/20130010174.

[4] Magee, T. E., Wilcox, P. A., Fugal, S. R., Acheson, K. E., Adamson, E. E., Bidwell, A. L., and Shaw, S. G., "System-Level Experimental Validations for Supersonic Commercial Transport Aircraft Entering Service in the 2018-2020 Time Period," NASA CR-2013-217797, NASA Langley Research Center, Feb. 2013. doi:2060/20130011026.

[5] Yoshida, K., "Supersonic drag reduction technology in the scaled supersonic experimental airplane project by JAXA,” Progress in Aerospace Sciences, Vol. 45, No. 4, 2009, pp. 124-146.

[6] Park, M. A., Aftosmis, M. J., Campbell, R. L., Carter, M. B., Cliff, S. E., and Bangert, L. S., "Summary of the 2008 NASA Fundamental Aeronautics Program Sonic Boom Prediction Workshop,' Journal of Aircraft, 2014. URL http: $/ / \mathrm{dx}$.doi.org/10.2514/1.CQ32589

[7] Park, M. A., and Morgenstern, J. M., "Summary and Statistical Analysis of the First AIAA Sonic Boom Prediction Workshop," AIAA Paper 2014-2006, 2014.

[8] Park, M. A., and Nemec, M., "Near Field Summary and Statistical Analysis of the Second AIAA Sonic Boom Prediction Workshop," AIAA Paper 2017-3256, 2017.

[9] Aftosmis, M., Nemec, M., and Cliff, S., “Adjoint-based low-boom design with Cart3D,” AIAA Paper 2011-3500, 2011. 
[10] Nemec, M., and Aftosmis, M. J., "Parallel adjoint framework for aerodynamic shape optimization of component-based geometry," AIAA Paper 2011-1249, 2011.

[11] Rallabhandi, S., Nielsen, E., and Diskin, B., "Sonic-Boom Mitigation Through Aircraft Design and Adjoint Methodology," Journal of Aircraft, Vol. 51, No. 2, 2014, pp. 502-510. doi:10.2514/1.C032189.

[12] Ordaz, I., and Li, W., "Adaptive Aft Signature Shaping of a Low-Boom Supersonic Aircraft Using Off-Body Pressures,” AIAA Paper 2012-0020, 2012.

[13] Silva, W. A., Sanetrik, M. D., Chwalowski, P., Connolly, J., and Kopasakis, G., "Using FUN3D for Aeroelastic, Sonic Boom, and AeroPropulsoServoElastic (APSE) Analyses of a Supersonic Configuration,” AIAA Paper 2016-1319, 2016.

[14] West IV, T. K., Reuter, B. W., Walker, E. L., Kleb, B., and Park, M. A., "Uncertainty Quantification and Certification Prediction of Low-Boom Supersonic Aircraft Configurations," Journal of Aircraft, Vol. 54, No. 1, 2017, pp. 40-53.

[15] Ng, L. W. T., and Eldred, M. S., "Multifidelity Uncertainty Quantification Using Non-Intrusive Polynomial Chaos and Stochastic Collocation,” AIAA Paper 2012-1852, 2012.

[16] West IV, T. K., and Gumbery, C., "Multifidelity, Multidisciplinary Design Under Uncertainty with Non-Intrusive Polynomial Chaos," AIAA Paper 2017-1936, 2017.

[17] Oberkampf, W., Helton, J., and Sentz, K., "Mathematical representation of uncertainty,” AIAA Paper 2001-1645, 2001.

[18] Hosder, S., and Bettis, B., "Uncertainty and sensitivity analysis for reentry flows with inherent and model-form uncertainties," Journal of Spacecraft and Rockets, Vol. 49, No. 2, 2012, pp. 193-206.

[19] Bettis, B., Hosder, S., and Winter, T., "Efficient Uncertainty Quantification in Multidisciplinary Analysis of a Reusable Launch Vehicle," AIAA Paper 2011-2393, 2011.

[20] Xiu, D., and Karniadakis, G. E., “The Wiener-Askey Polynomial Chaos for Stochastic Differential Equations,” SIAM Journal on Scientific Computing, Vol. 24, No. 2, 2002, pp. 619-644.

[21] Eldred, M. S., "Recent Advances in Non-Intrusive Polynomial Chaos and Stochastic Collocation Methods for Uncertainty Analysis and Design,” AIAA Paper 2009-2274, 2009, May 2009.

[22] Ghanem, R. G., and Spanos, P. D., Stochastic Finite Elements: A Spectral Approach, Springer-Verlag, New York, 1991.

[23] West IV, T. K., Hosder, S., and Johnston, C. O., "Multi-Step Uncertainty Quantification Approach Applied to Hypersonic Reentry Flows," Journal of Spacecraft and Rockets, Vol. 51, No. 1, 2014, pp. 296-310.

[24] Lockwood, B., and Mavriplis, D., “Gradient-Based Methods for Uncertainty Quantification in Hypersonic Flows,” Computers and Fluids Journal, Vol. 85, 2013, pp. 27-38.

[25] Roderick, O., Anitescu, M., and Fischer, P., "Polynomial Regression Approaches Using Derivative Information for Uncertainty Quantification,” Nuclear Science and Engineering, Vol. 164, No. 2, 2010, pp. 122-139. 
[26] Hosder, S., Walters, R. W., and Balch, M., "Point-Collocation Nonintrusive Polynomial Chaos Method for Stochastic Computational Fluid Dynamics,” AIAA Journal, Vol. 48, No. 12, 2010, pp. 2721-2730.

[27] Han, D., and Hosder, S., "Inherent and Model-Form Uncertainty Analysis for CFD Simulation of Synthetic Jet Actuators," AIAA Paper 2012-0082, 2012, Jan. 2012.

[28] Hosder, S., Walters, R. W., and Balch, M., "Efficient Sampling for Non-Intrusive Polynomial Chaos Applications with Multiple Uncertain Input Variables,” AIAA Paper 2007-0125, 2007.

[29] Biedron, R. T., and et al., “FUN3D Manual: 13.1,” Tech. rep., NASA/TM-2017-219580, Feb. 2017.

[30] Shah, H. R., Hosder, S., and Winter, T., “A Mixed Uncertainty Quantification Approach with Evidence Theory and Stochastic Expansions,” AIAA Paper 2014-0298, 2014, 2014.

[31] Schaefer, J., Hosder, S., West, T., Rumsey, C., Carlson, J., and Kleb, W., "Uncertainty Analysis of Turbulence Model Closure Coefficients for Transonic Wall-Bounded Flows," AIAA Journal, Vol. 55, No. 1, 2017, pp. 195-213.

[32] Erb, A., and Hosder, S., "Uncertainty Analysis of Turbulence Model Closure Coefficients for Wall-Bounded Attached and Separated Flows,” AIAA Paper 2017-1952, 2017.

[33] Erb, A., and Hosder, S., "Uncertainty Analysis of Turbulence Model Closure Coefficients for Shock Wave-Boundary Layer Interaction Simulations,” AIAA Paper 2018-2077, 2018.

[34] Palacios, F., Alonso, J. J., Colonno, M., Hicken, J., and Lukaczyk, T., “Adjoint-Based Method for Supersonic Aircraft Design Using Equivalent Area Distributions,” AIAA Paper 2012-269, 2012.

[35] Rallabhandi, S. K., "Application of Adjoint Methodology to Supersonic Aircraft Design Using Reversed Equivalent Areas," Journal of Aircraft, Vol. 51, No. 6, 2014, pp. 1873-1882.

[36] Palacios, F., Alonso, J. J., Colonno, M., Hicken, J., and Lukaczyk, T., “Adjoint-based method for supersonic aircraft design using equivalent area distribution,” AIAA Paper 2012-0269, 2012.

[37] Li, W., and Rallabhandi, S., "Inverse Design of Low-Boom Supersonic Concepts Using Reversed Equivalent-Area Targets," Journal of Aircraft, Vol. 51, No. 1, 2014, pp. 29-36.

[38] Park, M. A., Aftosmis, M. J., Campbell, R. L., Carter, M. B., Cliff, S. E., and Bangert, L. S., "Summary of the 2008 NASA Fundamental Aeronautics Program Sonic Boom Prediction Workshop,” AIAA, 2013, 2013.

[39] Aftosmis, M. J., Nemec, M., and Wintzer, M., "Sonic-Boom Prediction with Output-Based Adaptation and Cart3D," October 2008, October 2008.

[40] Phillips, B. D., and West, T. K., “Trim Flight Conditions for a Low-Boom Aircraft Design Under Uncertainty,” AIAA Paper 2017-3047, 2017. 
[41] Phillips, B. D., and West, T. K., “Aeroelastic Uncertainty Quantification of a Low-Boom Aircraft Configuration,” AIAA Paper 2017-0333, 2018.

[42] Rallabhandi, S. K., “Advanced Sonic Boom Prediction Using the Augmented Burgers Equation,” Journal of Aircraft, Vol. 48, No. 4, 2011, pp. 1245-1253. doi:10.2514/1.C031248.

[43] Shepherd, K. P., and Sullivan, B. M., “A Loudness Calculation Procedure Applied to Shaped Sonic Booms,” Tech. Rep. 3134, NASA, November 1991.

[44] Rallabhandi, S., and Loubeau, A., "Propagation Summary of the Second AIAA Sonic Boom Prediction Workshop," AIAA Paper 2017-3257, 2017, jan 2017. doi:12.1.

[45] Schwartz, B., and Benjamin, S. G., "A Comparison of Temperature and Wind Measurements from ACARS-Equippped Aircraft and Rawinsondes," Weather and Forecasting, Vol. 10, 1995, pp. 528-544.

[46] Sudret, B., "Global sensitivity analysis using polynomial chaos expansion,” Reliability Engineering and System Safety, Vol. 93, No. 7, 2008, pp. 964-979. 